\title{
Large-scale functionalization of biomedical porous titanium scaffolds surface with $\mathrm{TiO}_{2}$ nanostructures
}

\author{
Lei Qian ${ }^{1,2 \dagger}$, Peng $\mathrm{Yu}^{1,2 \dagger}$, Jinquan Zeng ${ }^{3}$, Zhifeng Shi ${ }^{1,2}$, Qiyou Wang ${ }^{4 *}$, Guoxin Tan ${ }^{5}$ and \\ Chengyun Ning ${ }^{1,2^{*}}$
}

\begin{abstract}
Construction of functional porous titanium scaffold is drawing ever growing attention, due to its effectiveness in solving the mechanical mismatch between titanium implant and bone tissue. However, the poor water permeability as well as the problem in achieving uniform surface modification inside scaffold hinders the further biomedical application of porous titanium scaffold. In this study, largescale functional $\mathrm{TiO}_{2}$ nanostructures (nanonetwork, nanoplate and nanowire) were constructed on three-dimensional porous titanium scaffolds surface via an effective hydrothermal treatment method. These nanostructures increase the hydrophilicity of the titanium scaffold surface, facilitating the cell culture medium to penetrate into the inner pore of the scaffold. Zeta potential analyses indicate that the surface electrical properties depend on the nanostructure, with nanowire exhibiting the lowest potential at $\mathrm{pH}$ 7.4. The influence of the nano-functionalized scaffold on protein adsorption and cell adhesion was examined. The results indicate that the nano-functionalized surface could modulate protein adsorption and bone marrow derived mesenchymal stem cells (BMSCs) adhesion, with the nanowire functionalized porous scaffold homogeneously promoting protein adsorption and BMSCs adhesion. Our research will facilitate future research on the development of novel functional porous scaffold.
\end{abstract}

Keywords: porous titanium, hydrothermal, nanostructure, scaffold, cell adhesion

\section{INTRODUCTION}

Titanium and its alloys are promising materials for dental implants and repairing severe skeletal damage caused by tumor and trauma $[1,2]$. However, bulk titanium remains difficult to be used in bone tissue repair not only because of its poor Osteogenic properties but also its inappropriate elastic modulus for bone tissue and less space for bone cells to grow inside $[3,4]$. To solve the mismatching in mechanical properties, three-dimensional porous titanium scaffolds were proposed to be built to substitute bulk titanium as bone implant [5].

Although these porous titanium scaffolds could improve the mechanical mismatching, the bio-inert properties and poor solution permeability still limit the bone-implant related application of the titanium scaffold [6-8]. In most cases, the pore sizes of the hydrophobic porous titanium scaffolds are in the range of micrometer scale, which hinders the water solution from entering the inner part of the scaffold, resulting in the obstacle in cell permeability and substance exchanges. Thus, altering the surface wettability and bioactivity of the scaffold is the key to achieve effective porous titanium scaffold development.

In order to improve the surface functionality of the titanium and its alloy, various methods, such as sand blasting, electrochemical anodization and plasma spraying, were carried out to build bioactive structures on their surface $[9,10]$. However, the above methods are not suitable for porous titanium scaffolds surface modification because these methods are difficult to homogeneously modify the complex micro-structured scaffolds. Thus, it is still a challenge to functionalize porous titanium scaffold surface effectively and uniformly.

In addition to the above methods, hydrothermal treat-

\footnotetext{
${ }^{1}$ School of Materials Science and Engineering, South China University of Technology, Guangzhou 510641, China

${ }^{2}$ Biomedical Engineering Key Laboratory of Guangdong Province, Guangzhou 510641, China

${ }^{3}$ Guangzhou Tieyi Middle School, Guangzhou 510660, China

${ }^{4}$ Department of Spine Surgery, the Third Affiliated Hospital of Sun Yat-sen University, Guangzhou 510630, China

${ }^{5}$ School of Chemical Engineering and Light Industry, Guangdong University of Technology, Guangzhou 510006, China

${ }^{\dagger}$ These two authors contributed equally to this paper.

* Corresponding authors (emails: imcyning@scut.edu.cn (Ning C); wqiyou@163.com (Wang Q))
} 
ment remains a promising method to construct large-scale nanostructures at relative low temperature and low cost [11-13]. Hydrothermal treatment has already been used to bioactivate the titanium scaffold surface with functionalized nanostructures [14]. During hydrothermal treatment, the hot aqueous alkali enters the inner pores of the scaffolds, which ensures the uniform distribution of the nanostructures on the surface of the scaffold. Wu, et al. [14] built nanobelts film on a microporous orthopedic $\mathrm{NiTi}$ /Ti scaffold via a facile low temperature hydrothermal treatment. In another study, the hydrothermally synthesized nanoporous film was shown to promote new bone formation within the pores of the scaffold compared to the nanostructure prepared by anodization [8]. However, the limited hydrothermal reaction condition of these experiments may hinder further improvement of the osseointegrative properties of the hydrothermally treated titanium scaffold, because the topographical feature of the hydrothermally synthesized nanostructures fabricated on porous titanium scaffolds could present surrounding cells with different mechanotransductive cues to modulate cell behaviors $[15,16]$.

In this study, large-scale uniform nanostructures with different topographical features were constructed on rapid prototyped titanium scaffolds via controlling the hydrothermal reaction temperature. The composition, electrical and water permeable properties of these nano-functionalized scaffolds were examined. Studies were also performed to evaluate the relationship between protein adsorption and cell adhesion performance of these nanofunctionalized scaffolds.

\section{EXPERIMENTAL SECTION}

\section{Construction of porous titanium scaffolds}

Cylinder samples ( $\Phi 8 \mathrm{~mm} \times 5 \mathrm{~mm}$ ) which possess pore size ranging from 800 to $1000 \mu \mathrm{m}$ and strut thickness of 300 $\mu \mathrm{m}$ were designed using Magics 11 software (Materialise, Belgium). The designed three dimensional porous titanium scaffold was fabricated by using precommercial Dimetal-280 Selective Laser Melting facility (SLM, South China University of Technology, China) with the same optimized parameters as the previous research [17].

\section{Preparation of the nanostructures on scaffold surface}

These rapid prototyped scaffolds were ultrasonically cleaned in acetone and ethanol, respectively, and acid etched with 1:1 $(v / v) \mathrm{HF}$ and $\mathrm{HNO}_{3}$ solution for $10 \mathrm{~min}$. The nanostructures on porous titanium scaffolds were fabricated in $2 \mathrm{~mol} \mathrm{~L}^{-1} \mathrm{NaOH}$ solution (analysis reagent
(AR), Aladdin) in Teflon autoclave for $24 \mathrm{~h}$. The reaction temperatures were 60,100 and $140^{\circ} \mathrm{C}$, respectively. Subsequently, the samples were placed in a $1 \mathrm{mmol} \mathrm{L}{ }^{-1} \mathrm{HNO}_{3}$ solution for $24 \mathrm{~h}$. Finally, the samples were air-heat treated at $500^{\circ} \mathrm{C}$ for $2 \mathrm{~h}$.

\section{Characterization of the scaffold and nanostructures}

A scanning electron microscope (SEM, ZEISS EVO 18, Germany) equipped with an energy dispersive spectrometer (EDS) system (INCA 200, Oxford) was used to characterize the morphology and composition of the nanostructures. Raman spectroscopy (LabRAM HR800, HORIBA jobin Yvon, France) was used to characterize the phase composition of different nanostructures. Water permeability of nano-functionalized scaffold was examined by detecting the water contact angle using contact angle meter (DSA25, Kruss, Germany). $2 \mu \mathrm{L}$ volume of water and Dulbecco's modified eagle medium (DMEM) containing $10 \%$ fetal bovine serum (FBS) was dropped on the upper surface of scaffold at ambient temperature, respectively.

Zeta potentials of the acid etched titanium, nanonetwork, nanoplate and nanowire functionalized bulk titanium samples were detected using zetameter (Anton Paar SurPass, Germany) in $10^{-3} \mathrm{~mol} \mathrm{~L}^{-1} \mathrm{KCl}$ solution. For statistical reasons, four streaming potentials were measured at each $\mathrm{pH}$ value. The mean value of three data was used to calculatethe potential/pH function.

\section{Protein adsorption on the nano-functionalized scaffold surface}

Bovine serum albumin (BSA, Sigma, purity level: 99.8\%) and human fibronectin (FN, Gene operation, purity: $\geq 90 \%$ ) were used to examine the protein adsorption properties of acid etched, nanonetwork, nanoplate and nanowire functionalized porous scaffolds. For BSA adsorption assay, BSA was dissolved in phosphate buffer solution (PBS, Hyclone) to a final concentration of $1 \mathrm{mg} \mathrm{mL} \mathrm{m}^{-1}$. $400 \mu \mathrm{L}$ protein solution was pipetted onto sample surface, the plate was incubated at $37^{\circ} \mathrm{C}$ for $4 \mathrm{~h}$. Then the remaining protein solution was removed, and washed for three times. $400 \mu \mathrm{L} 1 \%$ sodium dodecyl sulfate (SDS, Sigma) was added to each well to elute the adherent protein at $37^{\circ} \mathrm{C}$ overnight. The concentration of protein was determined by using a micro Bicinchoninic Acid assay kit (BCA, Thermo) according to the manufacturer's instructions. FN adsorption assay was carried out in 100 $\mu \mathrm{g} \mathrm{mL}^{-1} \mathrm{FN}$ solution using the same method. The analysis was performed by using a spectral scanning multimode reader (Varioskan Flash, Thermo Scientific) at $562 \mathrm{~nm}$. 
The standard curve of the protein was obtained according to the guidance. When it came to statistical analysis, oneway ANOVA analysis of variance was used to identify significant differences (significance threshold: ${ }^{*} p<0.05$, $\left.{ }^{* *} p<0.01\right)$.

\section{Cell culture and cellular morphology observations}

The nano-functionalized scaffolds were autoclave sterilized. Mouse bone marrow derived mesenchymal stem cells (BMSCs, ATCC, USA) of passage 4 were used for the cell assay. BMSCs were seeded in 48-well plates in normal growth media (10\%FBS+DMEM, Gibco) at a density of 10,000 cells/well. The samples with the seeded cells were placed in a $37^{\circ} \mathrm{C}$ incubator under $5 \% \mathrm{CO}_{2}$.

SEM was applied to observe the spreading morphology of BMSCs. Cells were fixed on the scaffolds with $2.5 \%$ glutaraldehyde for $2 \mathrm{~h}$, then rinsed with PBS three times; dehydrated with a graded series of ethanol (30\%-100\%) and dried in a critical point dryer, coated with platinum and examined under SEM at $10 \mathrm{kV}$.

\section{RESULTS AND DISCUSSION}

In this research, we used SLM facility to fabricate cylindrical porous titanium scaffolds $(8 \mathrm{~mm}$ diameter and $5 \mathrm{~mm}$ height). The unit cell of the porous scaffold was designed as regular octahedral lattice to facilitate the Additive Manufacturing process [18]. Without addition of any support structure, the porous titanium scaffolds with highly interconnected pore structures were fabricated using the SLM facility (Fig. 1). Fig. 1a, b shows the top and side view of the scaffold, and the results indicate that the strut thickness is only about $30 \mu \mathrm{m}$ deviated from the

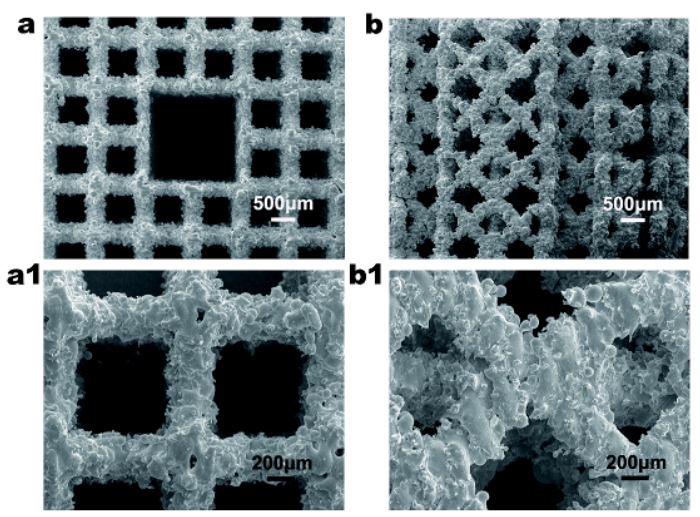

Figure 1 SEM image of the porous titanium scaffold prepared via selective laser melting technology: (a) and (a1) Top views of the scaffold, (b) and (b1) side views of the scaffold. Porous bone tissue engineering scaffold with interconnected pore structure was constructed based on the designed model. And the strut thickness of the scaffold is slightly deviated from the designed $300 \mu \mathrm{m}$ thickness. designed $300 \mu \mathrm{m}$ thickness. This interconnected porous scaffold could not only provide more space for newly formed bone tissue to connect with the scaffold, but also facilitate blood vessel and nerve formation [3,19]. The acid etching treatment was taken to wipe off the oxide layer on the surface of the porous titanium scaffold. The acid etched titanium scaffold surface shows micro-scale steplike structures (Fig. 2a, al). During the acid etching process, the titanium atoms at the grain boundary was preferentially etched due to the potential difference between grain boundaries and grains. The different etching velocity between titanium grain boundaries and grains results in the micro-scale step-like structures on the porous scaffold surface.

To achieve large-scale nanostructures construction on the titanium scaffold, hydrothermal treatments were carried out. The nanostructures were prepared via hydrothermal synthesis, during which the $\mathrm{TiO}_{2}$ nanostructures
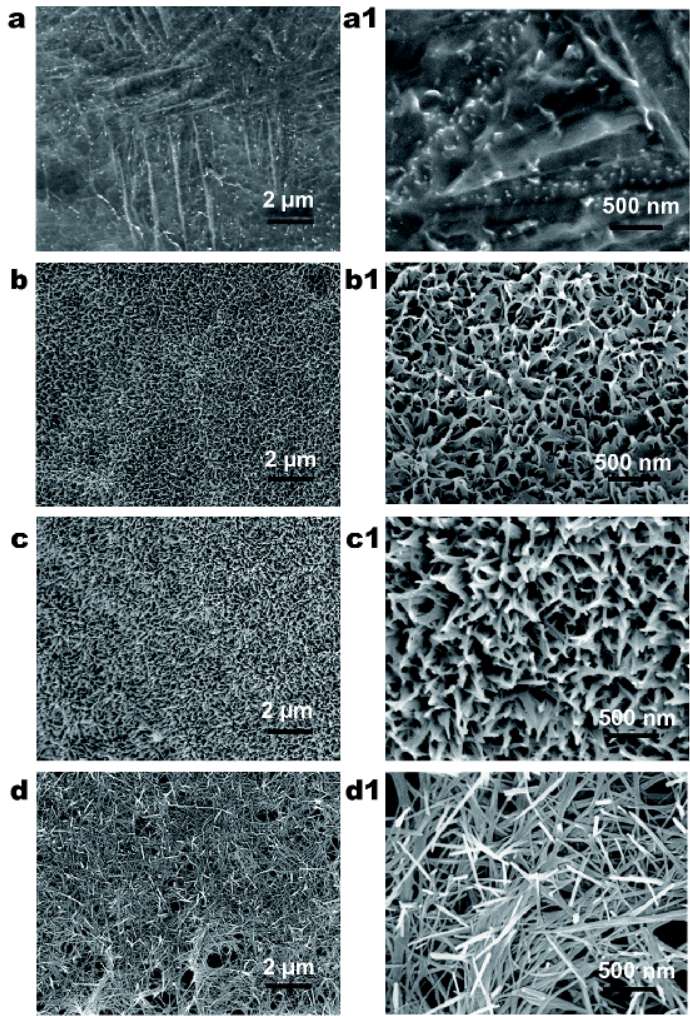

Figure 2 SEM image of the nanostructures on the porous titanium scaffold prepared at different hydrothermal temperature at the following acid immersion and heat treatment: (a) and (al) acid etched titanium scaffold; (b) and (b1) nanonetwork structure prepared at $60^{\circ} \mathrm{C}$; (c) and (c1) nanoplate structure prepared at $100^{\circ} \mathrm{C}$; (d) and (d1) nanowire structure prepared at $140^{\circ} \mathrm{C}$. The results indicate large-scale nanostructured film could be constructed on titanium surface, and that the morphology of the nanostructures could be dictated by the applied hydrothermal temperature. 
were constructed on the titanium scaffold surface through a dissolution recrystallization process, and the product nanotopography could be dominated by the reaction temperature $[20,21]$.

When the reaction temperature increased, more titanium precursor would dissolve, resulting in more nuclei formation on the surface of the titanium scaffolds. Meanwhile, higher temperature speeded up the recrystallization process, resulting in a higher nanostructures growth rate $[20,21]$. Thus, the nanostructures fabricated on the porous titanium scaffold surface could be tuned via controlling the hydrothermal temperature. With the increase of the hydrothermal treatment temperature from 60,100 to $140^{\circ} \mathrm{C}$, large-scale and uniform nanonetwork, nanoplate and nanowire films could be fabricated on the porous titanium scaffold respectively (Fig. 2).

EDS spectra (Fig. 3a) of the nanostructures indicate that all the nanostructures are mainly composed by titanium and oxygen element after the following acid immersion and heat treatment. Raman spectra (Fig. 3b) indicate that all the nanostructures are composed by anatase and rutile
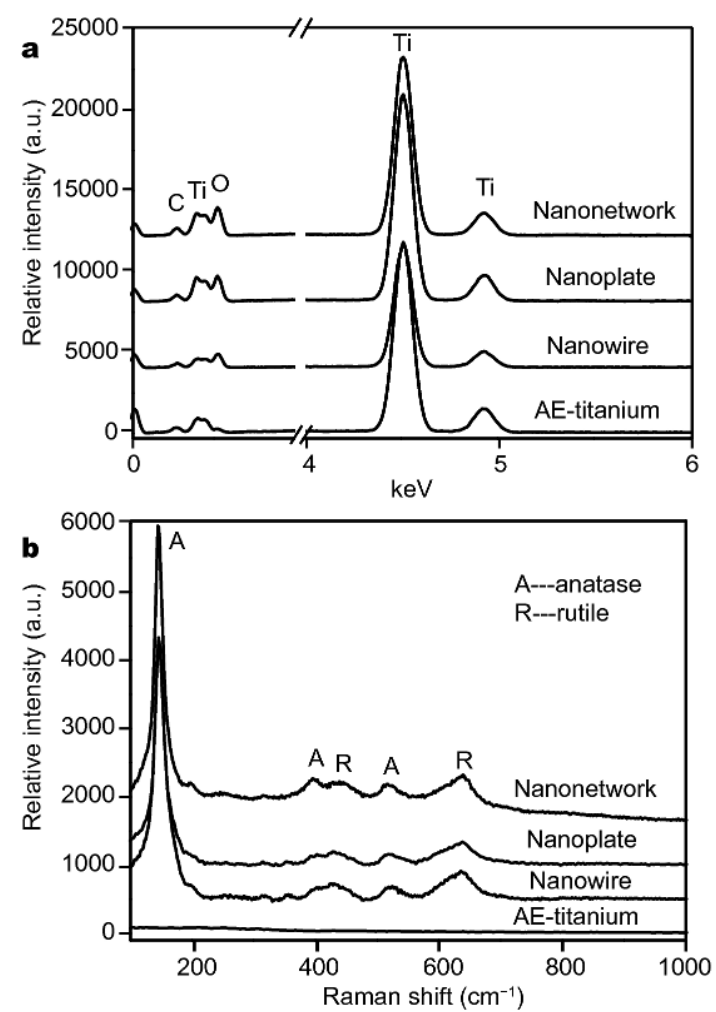

Figure 3 Element and phase composition of the acid etched, nanonetwork, nanoplate, and nanowire structures on titanium scaffolds surface. The results indicate that the compositions of the nanostructures built at different hydrothermal temperature on scaffolds are all converted to $\mathrm{TiO}_{2}$ (complex of anatase and rutile).
$\mathrm{TiO}_{2}$. The peaks at 146 and $637 \mathrm{~cm}^{-1}$ were ascribed to the symmetric stretching vibration of $\mathrm{O}-\mathrm{Ti}-\mathrm{O}$ in $\mathrm{TiO}_{2}$, and peaks at 396 and $515 \mathrm{~cm}^{-1}$ were assigned to the symmetric bending vibration and antisymmetric bending vibration of $\mathrm{O}-\mathrm{Ti}-\mathrm{O}$, respectively. The peaks at 146,396 and $515 \mathrm{~cm}^{-1}$ were ascribed to the anatase $\mathrm{TiO}_{2}$, and the other two peaks at 441 and $637 \mathrm{~cm}^{-1}$ were ascribed to the rutile $\mathrm{TiO}_{2}$. Various previous studies indicated that the morphology of the nanotopographical features could affect the cellular differentiation process [15].

To mimic the in vivo body fluid penetration into the nano-functionalized titanium scaffolds, contact angle of the ultrapure water and DMEM solution was taken using a contact angle meter. As shown in Fig. 4a, al, both water and DMEM solution could not penetrate into the acid etched titanium scaffold, and their contact angles were $112.5 \pm 5.4^{\circ}$ and $122.0 \pm 8.3^{\circ}$, respectively. These results mean that the acid etched titanium scaffold is hydrophobic, making the water solution difficult to penetrate into the inner surface, which is in accordance with previous reports $[8,22]$, which will hinder its application as bone implants. The nanostructures constructed on the scaffold surface via hydrothermal treatment increase the specific surface area of the scaffold and thus alter the wettability of the scaffold. Both water and DMEM solution would spread along the nano-functionalized scaffold surface to penetrate into the inner surface of the scaffold, which is supposed to facilitate cells and nutrition exchanges (Fig. 4).

The surface electrical cues conveyed by the $\mathrm{TiO}_{2}$ nanostructures were demonstrated to affect protein adsorption and stem cell behaviors [23]. To evaluate the surface electrical properties of the nanostructured films, zeta potential, the potential between the Stern layer and the dif-
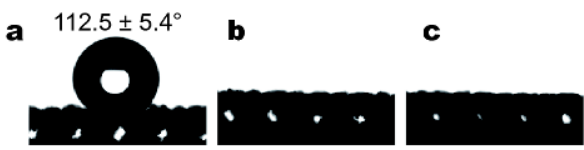

d
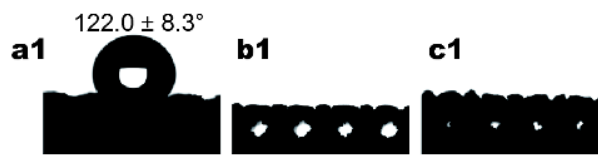

d1

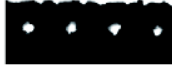

Figure 4 Water permeability of the nano-functionalized scaffolds examined via contact angle analysis. (a-d) Water contact angle analysis of (a) acid etched titanium, (b) nanonetwork, (c) nanoplate and (d) nanowire. (a1-d1) Growth media contact angle analysis of (a1) the acid etched, (b1) nanonetwork, (c1) nanoplate and (d1) nanowire. These results indicate that these $\mathrm{TiO}_{2}$ nanostructures improve the water solution permeability of the porous titanium scaffold, which would facilitate cells and nutrition exchanges. 
fuse layer, was detected on bulk samples constructed with the same selective laser melting parameters and hydrothermal conditions [24]. When the nano-functionalized samples were immersed into an aqueous solution, its surface would immediately cover by hydroxyl groups [25]. And due to their different specific surface areas, the amount of formed hydroxyl groups would vary among the nanonetwork, nanoplate and nanowire functionalized samples. Different amount of hydroxyl groups would induce distinct unspecific adsorption of electrolyte ions on these nanostructures surface, which induced the zeta potential difference among these nanostructures [26]. The zeta potential measurement results suggest that the asprepared $\mathrm{TiO}_{2}$ nanostructures could convert the zeta potential of the titanium substrate, and the lowest zeta potential was obtained on the nanowire surface compared with the other nanostructures at $\mathrm{pH}>4.2$. At physiological condition ( $\mathrm{pH} 7.4$, illustrated with a dash line in Fig. 5), the lowest zeta potential was obtained on the nanowire group reaching approximately $-50 \mathrm{mV}$. These results indicate that the zeta potential of the samples is closely related to the $\mathrm{TiO}_{2}$ nanostructures.

In in vivo environment, the biological response to biomaterial is mediated by the adsorbed protein layers on the biomaterials surface. In order to examine the amount of protein adsorption on the nano-functionalized porous titanium surfaces, the samples were examined with FN and BSA, which are the representative cell adhesive protein and the model protein, respectively. The quantitative analysis using a BCA (Fig. 6) indicated that the nanofunctionalized porous titanium scaffold could promote both FN and BSA adsorption, with the nanowire func-

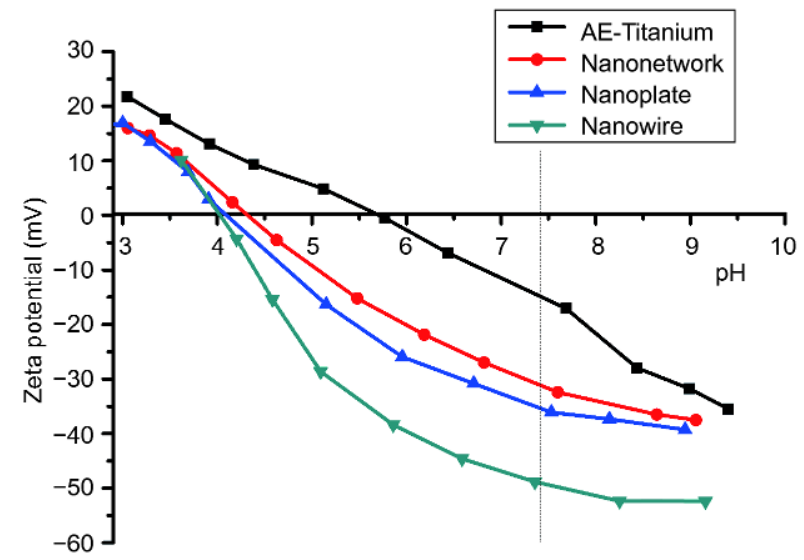

Figure 5 Zeta potential analyses of different nanostructured surfaces (pH 3-9) in $\mathrm{KCl}$ solution. The dash line shows the physiological condition ( $\mathrm{pH}$ 7.4). The results indicate that the zeta potential is distinct among different nanostructures.
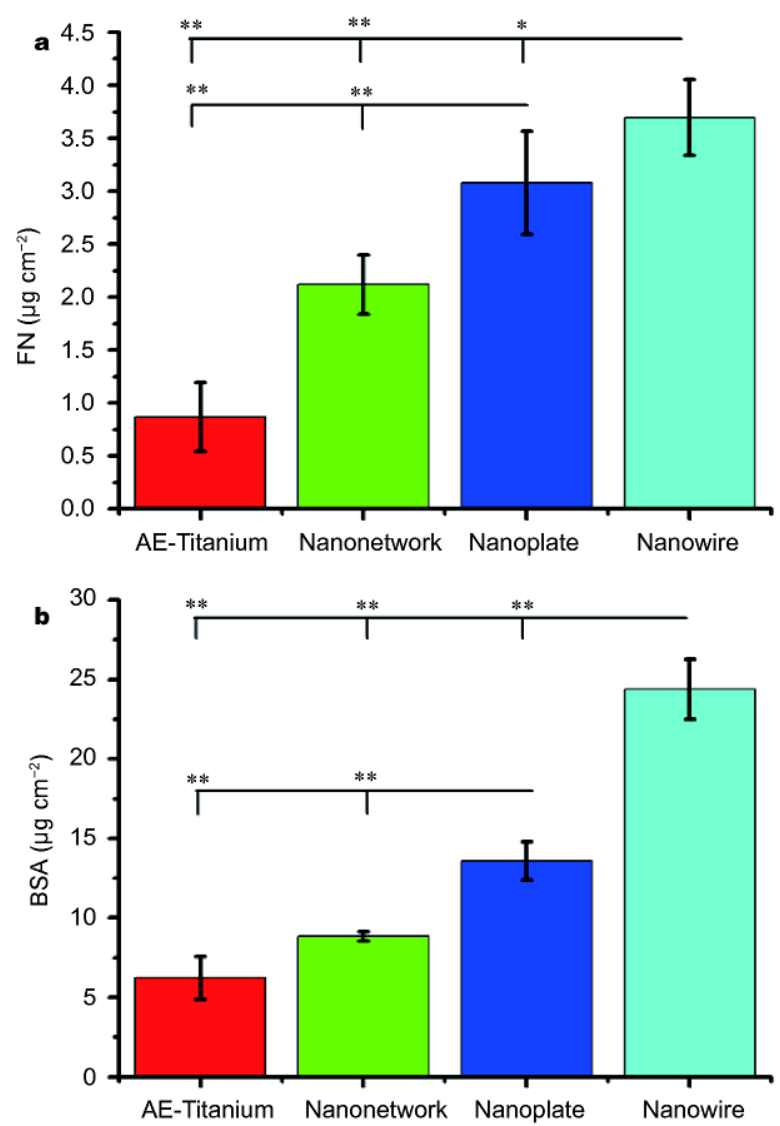

Figure $6 \mathrm{FN}$ and BSA protein adsorption on nano-functionalized scaffolds. The results indicate that the nanoplate and nanowire functionalized scaffolds could promote protein adsorption evidently. Error bars indicate a standard deviation for $n=6$. Each value is the mean value \pm standard deviation. ${ }^{*} p<0.05,{ }^{*} p<0.01, n=6$.

tionalized titanium scaffold achieving the highest adsorbed protein amount. Nearly 5 -fold increase in FN adsorption could be observed on nanowire sample compared to the acid etched porous titanium scaffold. Moreover, almost 4fold increase in BSA adsorption could be observed on nanowire sample compared to the acid etched porous titanium scaffold. The nanoplate shows moderate BSA and FN adsorption property. Thus, there is a positive correlation between protein adsorption and zeta potential (Fig. 5 ) as well as the aspect ratio of the nanostructures. And many researches demonstrated that the protein adsorbed on the nanostructures would mediate the cell nanotopographical interactions via the mechanotransductive cascade $[27,28]$.

To examine the cell adhesion on these nano-functionalized scaffolds, BMSCs were cultured on these functionalized scaffolds for $24 \mathrm{~h}$. The cells on the scaffolds surface were fixed and observed using SEM. The results indicate 
a
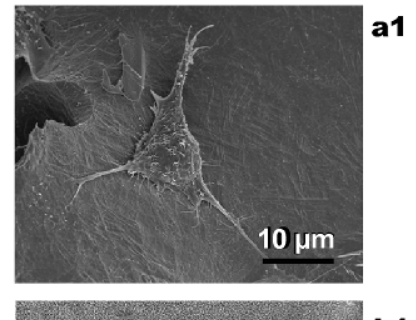

b
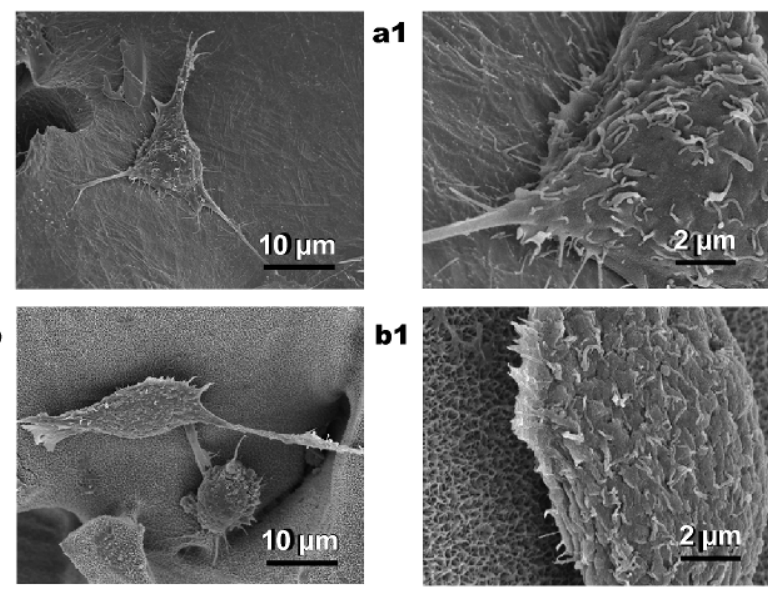

c

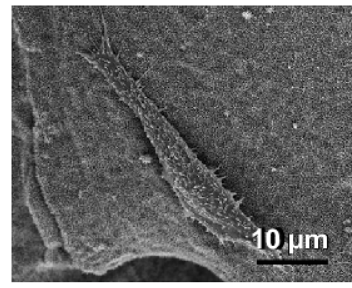

d

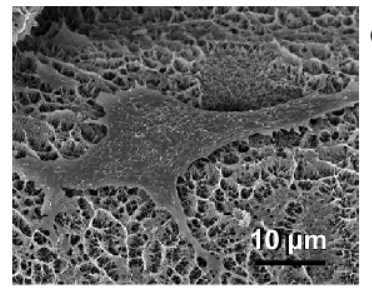

b1

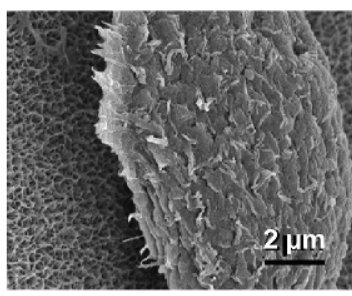

c1

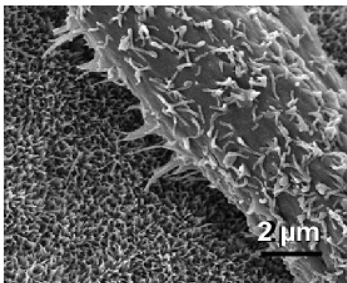

d1

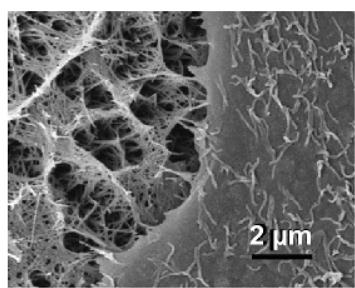

Figure 7 SEM micrographs of BMSCs cells cultured for $24 \mathrm{~h}$ on (a) and (a1) acid etched titanium scaffold, (b) and (b1) nanonetwork, (c) and (c1) nanoplate and (d) and (d1) nanowire functionalized titanium scaffold. The results indicate that the BMSCs adhere and proliferate well on the nanoplate and nanowire functionalized scaffolds compared to acid etched titanium and nanonetwork functionalized scaffold.

that the BMSCs attached well and became elongated on all the nano-functionalized porous titanium scaffolds, showing a spindle-shaped morphology (Fig. 7). Moreover, the cells on the nanowire functionalized scaffolds obtained a larger spreading area compared to the cells on the acid etched titanium scaffold. The magnified SEM images (Fig. 7a1-d1) show that the BMSCs attach to the scaffold surface via filopodia, and the bonding between the filopodia and the nanostructures would guide cells to perceive and respond to the nanotopographical cues $[29,30]$.

Previous studies have demonstrated that cell adhesion is closely related to the preadsorbed protein layers on the substrate surface $[13,28]$. In this study, the nanostructures constructed on the porous titanium scaffolds have been demonstrated to acquire different cell adhesive protein adsorption ability. Because the nanowire functionalized

scaffolds adsorbed more FN and BSA compared to the other nanostructures (Fig. 6a). The increasing protein adsorbed on nanowire structure may provide more sites for cells to adhere and spread. The hydrothermal treatment provided an easy and effective way to functionalize porous titanium scaffolds surface with large-scale nanostructures, which would be expected to modulate BMSCs behavior.

\section{CONCLUSIONS}

In this research, large-scale uniform nanonetworks, nanoplates and nanowire films were constructed on rapid prototyped porous titanium scaffolds surface through controlling the hydrothermal treatment temperature. These nanostructures were demonstrated to be mainly composed by anatase and rutile $\mathrm{TiO}_{2}$. The water solution permeability of the porous titanium scaffold was improved by the introduction of these nanostructures. Due to a large specific surface area, excellent solution permeability and relative lower Zeta potential, significant increase in FN and BSA adsorption were achieved on the nanowire-functionalized porous titanium scaffold compared to other porous titanium scaffolds, and further promote cell adhesion and spreading on the nanowire-functionalized scaffold. The research reveals that hydrothermal treatment is a convenient and effective way to functionalize porous titanium scaffold for biomedical application.

Received 11 April 2017; accepted 16 May 2017;

published online 28 June 2017

1 Geetha M, Singh AK, Asokamani R, et al. Ti based biomaterials, the ultimate choice for orthopaedic implants - a review. Prog Mater Sci, 2009, 54: 397-425

2 Liu X, Chu P, Ding C. Surface modification of titanium, titanium alloys, and related materials for biomedical applications. Mater Sci Eng-R-Rep, 2004, 47: 49-121

3 Bose S, Roy M, Bandyopadhyay A. Recent advances in bone tissue engineering scaffolds. Trends Biotech, 2012, 30: 546-554

4 Li JP, de Wijn JR, Van Blitterswijk CA, et al. Porous $\mathrm{Ti}_{6} \mathrm{Al}_{4} \mathrm{~V}$ scaffold directly fabricating by rapid prototyping: preparation and in vitro experiment. Biomaterials, 2006, 27: 1223-1235

5 Van Bael S, Kerckhofs G, Moesen M, et al. Micro-CT-based improvement of geometrical and mechanical controllability of selective laser melted $\mathrm{Ti}_{6} \mathrm{Al}_{4} \mathrm{~V}$ porous structures. Mater Sci Eng-A, 2011, 528: 7423-7431

6 Chai YC, Truscello S, Bael SV, et al. Perfusion electrodeposition of calcium phosphate on additive manufactured titanium scaffolds for bone engineering. Acta Biomater, 2011, 7: 2310-2319

7 Fan X, Feng B, Weng J, et al. Processing and properties of porous titanium with high porosity coated by bioactive titania nanotubes. Mater Lett, 2011, 65: 2899-2901

8 Amin Yavari S, van der Stok J, Chai YC, et al. Bone regeneration 
performance of surface-treated porous titanium. Biomaterials, 2014, 35: 6172-6181

9 Dohan Ehrenfest DM, Coelho PG, Kang BS, et al. Classification of osseointegrated implant surfaces: materials, chemistry and topography. Trends Biotech, 2010, 28: 198-206

10 Park J, Bauer S, Schlegel KA, et al. $\mathrm{TiO}_{2}$ nanotube surfaces: $15 \mathrm{~nm}$ an optimal length scale of surface topography for cell adhesion and differentiation. Small, 2009, 5: 666-671

11 Zhou W, Liu X, Cui J, et al. Control synthesis of rutile $\mathrm{TiO}_{2} \mathrm{mi}-$ crospheres, nanoflowers, nanotrees and nanobelts via acid-hydrothermal method and their optical properties. CrystEngComm, 2011, 13: 4557

12 Feng S, Xu R. New materials in hydrothermal synthesis. Acc Chem Res, 2001, 34: 239-247

13 Yu P, Zhu X, Wang X, et al. Periodic nanoneedle and buffer zones constructed on a titanium surface promote osteogenic differentiation and bone calcification in vivo. Adv Healthcare Mater, 2016, 5: 364-372

14 Wu S, Liu X, Hu T, et al. A biomimetic hierarchical scaffold: natural growth of nanotitanates on three-dimensional microporous Ti-based metals. Nano Lett, 2008, 8: 3803-3808

15 Bettinger CJ, Langer R, Borenstein JT. Engineering substrate topography at the micro- and nanoscale to control cell function. Angew Chem Int Ed, 2009, 48: 5406-5415

16 Dalby MJ, Gadegaard N, Tare R, et al. The control of human mesenchymal cell differentiation using nanoscale symmetry and disorder. Nat Mater, 2007, 6: 997-1003

$17 \mathrm{Yu} \mathrm{P,} \mathrm{Lu} \mathrm{F,} \mathrm{Zhu} \mathrm{W,} \mathrm{et} \mathrm{al.} \mathrm{Bio-inspired} \mathrm{citrate} \mathrm{functionalized} \mathrm{apatite}$ coating on rapid prototyped titanium scaffold. Appl Surf Sci, 2014, 313: 947-953

18 Su X, Yang Y, Yu P, et al. Development of porous medical implant scaffolds via laser additive manufacturing. Trans Nonferrous Met Soc China, 2012, 22: s181-s187

19 Wang J, Yang M, Zhu Y, et al. Phage nanofibers induce vascularized osteogenesis in 3D printed bone scaffolds. Adv Mater, 2014, 26: 4961-4966

20 Yu X, Wang H, Liu Y, et al. One-step ammoniahydrothermal synthesis of single crystal anatase $\mathrm{TiO}_{2}$ nanowires for highly efficient dye-sensitized solar cells. J Mater Chem A, 2013, 1: 21102117

21 Bavykin DV, Friedrich JM, Walsh FC. Protonated titanates and $\mathrm{TiO}_{2}$ nanostructured materials: synthesis, properties, and applications. Adv Mater, 2006, 18: 2807-2824

22 Rupp F, Scheideler L, Rehbein D, et al. Roughness induced dynamic changes of wettability of acid etched titanium implant modifications. Biomaterials, 2004, 25: 1429-1438

23 Ning C, Yu P, Zhu Y, et al. Built-in microscale electrostatic fields induced by anatase-rutile-phase transition in selective areas promote osteogenesis. NPG Asia Mater, 2016, 8: e243

24 Cai K, Frant M, Bossert J, et al. Surface functionalized titanium thin films: zeta-potential, protein adsorption and cell proliferation. Colloids Surf B-Biointerf, 2006, 50: 1-8

25 Kokubo T, Yamaguchi S. Bioactive Ti metal and its alloys prepared by chemical treatments: state-of-the-art and future trends. Adv Eng Mater, 2010, 12: B579-B591

26 Pedimonte BJ, Moest T, Luxbacher T, et al. Morphological zetapotential variation of nanoporous anodic alumina layers and cell adherence. Acta Biomater, 2014, 10: 968-974

27 Sousa SR, Brás MM, Moradas-Ferreira $\mathrm{P}$, et al. Dynamics of fibronectin adsorption on $\mathrm{TiO}_{2}$ surfaces. Langmuir, 2007, 23: 70467054

28 Ngandu Mpoyi E, Cantini M, Reynolds PM, et al. Protein adsorption as a key mediator in the nanotopographical control of cell behavior. ACS Nano, 2016, 10: 6638-6647

29 Variola F, Brunski JB, Orsini G, et al. Nanoscale surface modifications of medically relevant metals: state-of-the art and perspectives. Nanoscale, 2011, 3: 335-353

30 Padmanabhan J, Kinser ER, Stalter MA, et al. Engineering cellular response using nanopatterned bulk metallic glass. ACS Nano, 2014, 8: 4366-4375

Acknowledgements This work was supported by the National High Technology Research and Development Program of China (2015AA033502), the National Natural Science Foundation of China (51372087, 51232002 and 51541201), the Science and Technology Planning Project of Guangdong Province, China (2014A010105048), the Natural Science Foundation of Guangdong Province (2015A030313493 and 2016A030308014), the State Key Laboratory for Mechanical Behavior of Materials, China (20141607) and the Technological Projects of Guangzhou, China (201604020110).

Author contributions Qian $\mathrm{L}$ and $\mathrm{Yu} \mathrm{P}$ conceived and designed the experiments, analyzed the results, wrote the manuscript. Zeng J and Shi $\mathrm{Z}$ prepared the samples and participated the characterization, Tan $\mathrm{G}$ involved in the paper writing and experiments designing, Wang $\mathrm{Q}$ and Ning C supervised the experiments designing and paper writing. All authors contributed to the general discussion.

Conflict of interest The authors declare that they have no conflict of interest.

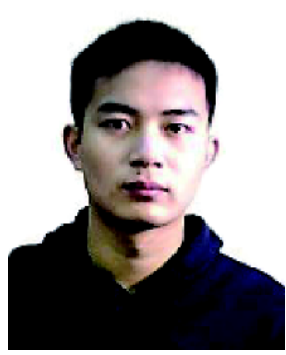

Lei Qian received his bachelor's degree from Wuhan Institute of Technology in 2013. Now he is pursuing his master's degree (biomedical engineering) at South China University of Technology. His research interests focus on the technique improving surface of metallic materials. 

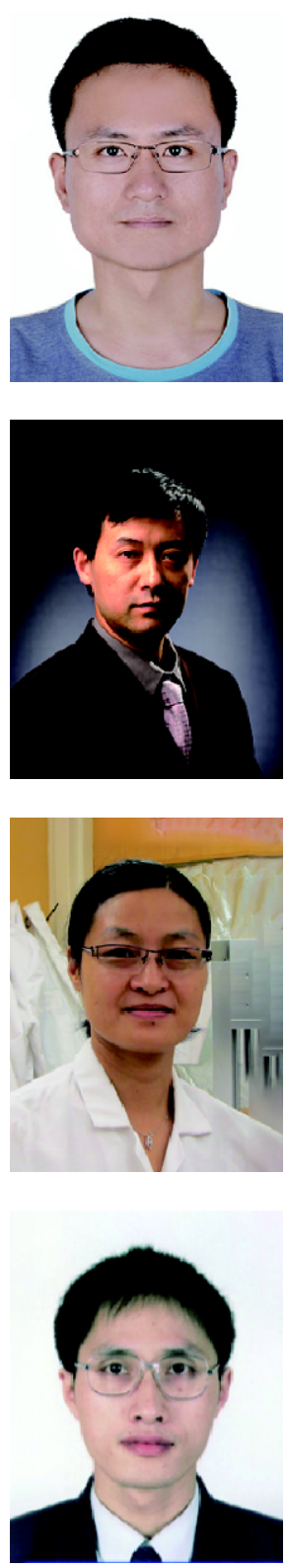

Peng Yu received his PhD degree in biomedical engineering from South China University of Technology in 2016. Now he is a postdoctoral researcher at the School of Materials Science and Engineering, South China University of Technology. His research interests focus on multiscale bone regenerative biomaterials, piezoelectric biomaterials and semi-conductive biomaterials.

Qiyou Wang is a professor of the Third Affiliated Hospital, Sun Yat-Sen University. Meanwhile, he is the secretary of Guangdong Tissue Engineering Society. His current research interests include stem cells and biomaterials for spinal cord injury and bone repair.

Guoxin Tan is currently a professor of the School of Chemical Engineering and Light Industry, Guangdong University of Technology. She received her PhD degree in biomaterials science from Guangdong University of Technology (China) in 2004. After postdoctoral training at South China University of Technology in 2006-2009, she also had an experience as a visiting scholar in Harvard Medical School/Brigham and Women's Hospital in 2010-2011. Her major research interests include surface modifications of implants and biopolymer-based hydrogel.

Chengyun Ning is currently a full professor in the School of Materials Science and Engineering, South China University of Technology. He is the Director of Biomedical Engineering Key Laboratory of Guangdong Province, China, the Principle Investigator of the National Basic Research Program (973). His major research interests include surface modifications of implants and electroactive biomaterials.

\section{医用多孔钠支架表面功能化二氧化钠纳米结构构建的研究}

钱否 ${ }^{1,2 \dagger}$, 于鹏 $^{1,2 \dagger}$, 曾锦全 ${ }^{3}$, 石志锋 $^{1,2}$, 王其友 ${ }^{*}$, 谭帼馨 ${ }^{5}$, 宁成云 ${ }^{1,2^{*}}$

摘要 由于可有效解决钛植入体与骨组织在力学方面的不匹配性, 功能化多孔钛支架的研发已得到广泛关注. 然而, 多孔钛支架往往液体渗透 性差且难以实现大面积均匀的表面处理, 极大阻碍了多孔支架用于临床骨缺损修复治疗. 本研究采用高效水热处理方法在三维多孔钛支架内 外表面构建均匀二氧化钛纳米结构(纳米网络结构、纳米片结构和纳米线结构). 这些纳米结构增强了多孔钛支架的亲水性, 利于细胞培养液 进入到多孔支架孔内. 固体表面Zeta电位分析表明材料表面电势依赖于材料表面纳米结构, 其中在 $\mathrm{pH}$ 为 7.4 条件下纳米线结构具有最低的表面 电势. 纳米功能化的多孔支架具有调控蛋白吸附和骨髓间充质干细胞黏附的作用, 其中纳米线结构功能化的多孔钛支架可同时促进蛋白吸附 和细胞黏附铺展. 该研究工作将对新型功能化医用多孔支架的研发具有指导意义. 